\title{
Criminologie
}

\section{Les médias : source de victimisation}

\section{Georges-André Parent}

Volume 23, numéro 2, 1990

Après le crime : survivre

URI : https://id.erudit.org/iderudit/017294ar

DOI : https://doi.org/10.7202/017294ar

Aller au sommaire du numéro

Éditeur(s)

Les Presses de l'Université de Montréal

ISSN

0316-0041 (imprimé)

1492-1367 (numérique)

Découvrir la revue

Citer cet article

Parent, G.-A. (1990). Les médias : source de victimisation. Criminologie, 23(2), 47-71. https://doi.org/10.7202/017294ar

\section{Résumé de l'article}

With the advent of the Bill of Rights, making the offender less accessible, journalists are falling back more than ever on the victim to feed their daily tales of victimization.

The author analyses the various forms this source of victimization takes; for some victims, they are generally crimes that are spectacular and violent, and are often perpetrated against the most susceptible and vulnerable victims.

Each type of media (radio, dailies, weeklies, television) represents a particular way of adding to the suffering of the victim, and each has its way of "exploiting" the victim. The victim becomes a tool of the media, both commercially and ideologically, often with the connivance of the police, who also uses the victim for its purposes.

The victim is portrayed in stereotype, according to the type of victimization reported and the offender implicated, creating a guilty or innocent victim, and literally depriving him of his own account of his victimization to make it an object of curiosity that sells well.

Finally, the author analyzes how the police and the media, by interaction, can exploit the victim under the pretext of prevention or crime control and even through certain phenomena such as the reporting of crime waves promoting fear of crime. The article concludes that the media should have more respect for victims of crime.
Tous droits réservés (C Les Presses de l'Université de Montréal, 1990
Ce document est protégé par la loi sur le droit d'auteur. L'utilisation des services d’Érudit (y compris la reproduction) est assujettie à sa politique d'utilisation que vous pouvez consulter en ligne.

https://apropos.erudit.org/fr/usagers/politique-dutilisation/ 
With the advent of the Bill of Rights, making the offender less accessible, journalists are falling back more than ever on the victim to feed their daily tales of victimization.

The author analyses the various forms this source of victimization takes; for some victims, they are generally crimes that are spectacular and violent, and are often perpetrated against the most susceptible and vulnerable victims.

Each type of media (radio, dailies, weeklies, television) represents a particular way of adding to the suffering of the victim, and each has its way of "exploiting" the victim. The victim becomes a tool of the media, both commercially and ideologically, often with the connivance of the police, who also uses the victim for its purposes.

The victim is portrayed in stereotype, according to the type of victimization reported and the offender implicated, creating a guilty or innocent victim, and literally depriving him of his own account of his victimization to make it an object of curiosity that sells well.

Finally, the author analyzes how the police and the media, by interaction, can exploit the victim under the pretext of prevention or crime control and even through certain phenomena such as the reporting of crime waves promoting fear of crime. The article concludes that the media should have more respect for victims of crime.

La victimologie «comme discours structuré sur les victimes et les victimisations» (Baril, 1984, p. 2) est née il y a près de 50 ans, avec Von Hentig, en 1948 (Fattah, 1980, p. 8), mais ce n'est que tout récemment que la recherche et le discours se sont détoumés un peu de l'infracteur pour se préoccuper davantage de la victimisation sous toutes ses formes, de ce qu'on a appelé la victimisation secondaire:

Le plus souvent, l'événement déborde le cadre d'une brève confrontation entre deux protagonistes. D'autres agences entrent sur scène dès que le

* Georges-André Parent, journaliste dans le domaine policier et judiciaire depuis 25 ans, termine sa maîtrise à l'École de criminologie de l'Université de Montréal. On peut le joindre au 217, rue de la Commune Ouest, bureau 210, Montréal, Québec, H2Y 3W8. 
rideau tombe sur le drame initial: hôpital, compagnie d'assurances, employeurs, presse, police, tribunaux.

(Baril, 1980, p. 101)

Les recherches victimologiques comme la victimologie en action ont surtout porté jusqu'à maintenant sur le traitement des victimes par la police et les tribunaux. La relation médias-victimes, comme objet d'étude et cadre d'intervention, n'en est encore qu'à ses tout débuts.

Le rôle de la victime de crime dans les médias a été analysé dans le cadre de recherches plus générales sur les représentations et la réaction sociale au crime, mais plus rarement dans les mécanismes intrinsèques de la relation médias-victimes, une interaction qui, croyons-nous, a sa propre dynamique.

On ne peut encore compter sur les chartes de droit et le processus judiciaire pour «civiliser» le traitement des victimes par les médias, et ainsi empêcher journalistes, bureaucraties de la nouvelle et entreprises commerciales de l'information d'utiliser la victime à leurs fins.

Dans les médias, avec l'émergence d'un droit nouveau garanti par les chartes, on a dû admettre que le droit à l' information et à la liberté d'expression n'étaient pas absolus (au Canada plus qu'aux États Unis) et devaient se conjuguer avec les droits fondamentaux de l'individu. Ce sont toutefois les droits de l'infracteur, surtout, qu'on a jusqu'à maintenant voulu garantir, rarement ceux de la victime.

Dans la construction des nouvelles comme dans le processus judiciaire, l'infracteur se retrouve avec plus de droits que la victime, ou du moins avec le pouvoir d'en forcer le respect.

Résultat: au moment où on commence à s'intéresser sérieusement au traitement des victimes de crimes par les médias, les médias se rabattent plus que jamais sur la victime pour alimenter la chronique policière et judiciaire, l'infracteur étant devenu plus difficilement accessible.

C'est pourquoi il nous a semblé pertinent de scruter cette relation médiasvictime, à travers 25 ans de pratique dans le traitement de la nouvelle.

Certaines victimes, d'une certaine criminalité, sont plus particulièrement les cibles et les instruments des médias (de certains médias plus que d'autres), en tant qu'entreprises commerciales et idéologiques elles-mêmes engagées dans une interaction avec d'autres agences de contrôle social.

Ce sont ces interactions, cette appropriation de la victime par les médias, sources de nouvelles victimisations, que nous tenterons d'explorer dans cet exposé. 
Nous décrirons dans un premier temps quelles sont les victimes qui font la manchette et comment chaque type de média (radio, quotidiens, hebdomadaires et télévision) peut représenter une source spécifique de victimisation.

À travers l'interaction médias-police, nous tenterons ensuite de cerner comment la victime devient la cible, l'enjeu, l'instrument des uns et des autres; certaines victimes plus que d'autres parce que servant mieux que d'autres les stratégies des entreprises de collecte et de traitement de l'information que sont les médias, en interaction avec les agences de contrôle pourvoyeuses de nouvelles.

Nous verrons comment on sélectionne les victimisations, comment se construit la nouvelle autour de la victime, et comment on présente cette victime, selon qu'on la définit innocente ou coupable.

Nous analyserons enfin deux phénomènes illustrant bien cette exploitation de la victime à travers la relation médias-police: la peur du crime et la construction des vagues de crimes.

\section{QUELLES VICTIMES?}

Nous croyons à l'instar de Baril (1984, pp. 256 à 269) que la définition de la victime devrait être assez large pour englober toute personne qui subit un préjudice du fait d'autrui, fait intentionnel ou non; la victime qui se perçoit comme telle, indépendamment des dommages objectifs; et les victimes d'actes autres que ceux que sanctionne le pénal.

La définition de la victime par les Nations Unies (1985, p. 3), bien que ne se référant qu'aux «actes ou omissions qui enfreignent les lois pénales en vigueur», décrit les préjudices subis comme «une atteinte à leur intégrité physique ou mentale, une souffrance morale, une perte matérielle, ou une atteinte grave à leurs droits fondamentaux...» (Ibid).

Dans notre analyse de la relation médias-victimes, nous traiterons surtout de la victime «comme une personne physique directement lésée dans ses droits fondamentaux par le fait volontaire d'autrui» (Baril 1985, p. 5) mais aussi de ses proches, d'autres personnes indirectement touchées.

De façon générale, enfin, les victimes et les victimisations qui font la nouvelle ne sont pas nécessairement représentatives, du moins en proportion, des victimes et des victimisations définies par le code et faisant l'objet d'enquêtes policières ou de procédures judiciaires.

Il n'y a que les victimes d'une certaine criminalité, spectaculaire et violente, qui tapissent les pages de faits divers. Et à l'intérieur de cette criminalité, 
on retrouvera surreprésentées certaines victimes, surtout des classes les plus pauvres et défavorisées. Cette sélection se fait tantôt avec et par la complicité d'autres agents de contrôle social; tantôt par les simples rouages des médias comme producteurs de nouvelles; tantôt dans un cadre idéologique; tantôt par simple intérêt mercantile, ces victimes étant facilement accessibles, manipulables et présentant peu de risques de poursuites judiciaires au lendemain d'un reportage un peu spectaculaire.

Certaines victimes, d'une certaine criminalité, sont surtout «exploitées» (le mot pris ici dans son sens le plus neutre), comme ce sont certains criminels (infracteurs définis criminels) qui sont surtout visibilisés par les médias. Et ce sont souvent des criminels et des victimes d'une même classe ou catégorie sociale. L'homicide, crime qui fait toujours la manchette, implique surtout un acteur et une victime qui se connaissent bien: $66 \%$ des cas dans l'étude de Wolfgang (1958); $64 \%$ pour Herjanic et Meyer (1976); $61 \%$ dans Mercy et al. (1986); 76,9\%, selon Statistique Canada, pour la période 1976 à 1985. Côté et Hodgins (1987), dans une recension des écrits sur l'homicide, n'ont découvert qu'un auteur, Wolfgang, qui a tenté de préciser le statut socioéconomique des homicidaires et des victimes: «Il postule néanmoins, écrivent Côté et Hodgins (p. 47), que l'acteur serait généralement issu de la classe sociale inférieure, alors que la victime serait d'un niveau social un peu plus élevé ou, pour le moins, de niveaux plus variables.»

Dans la mesure où les données américaines peuvent être transposées au Québec, on peut citer Conklin qui écrit, après avoir analysé l'ensemble des statistiques américaines et des enquêtes de victimisation:

The lower classes have a higher rate of victimization than the higher social classes for rape, robbery, aggravated assault, and theft from the person without contact. Rates of victimization also increase with decreasing income for assaultive violence with theft and for personal theft without injury, as well as for serious assaultive violence without theft.

(Conklin, 1981, p. 141)

Comme ce sont ces crimes spectaculaires qui sont surtout visibilisés par les médias, ce sont donc les victimes les plus vulnérables, les plus dépourvues, qui risquent de faire la manchette. À cela il faut évidemment excepter les crimes impliquant des victimes très connues du grand public dont la notoriété fera d'elles des cibles privilégiées pour les journalistes.

Le type de victime, aussi bien que le genre de victimisation, joueront donc un rôle important dans la sélection de la nouvelle et surtout dans son traitement. La contribution active ou passive de la victime, ses traits de personnalité, son mode de vie, son statut social, ses liens avec l'infracteur, les 
lieux et circonstances de l'événement détermineront la pertinence de la nouvelle, son importance et la façon dont on la traitera... quand ce ne sera pas tout simplement le fait pour le journaliste ou le photographe d'être au bon endroit au bon moment...!

\section{QUELS MÉDIAS?}

Comme ce ne sont pas toutes les victimes de crime qui alimentent la chronique policière et judiciaire, tous les médias ne traitent pas également et de la même façon les victimes.

Beaucoup d'auteurs éludent la problématique en se contentant de relier la victimisation secondaire par les médias à une certaine presse. Les journaux dits populaires, la presse spécialisée (par le contenu et non l'expertise) sont surtout visés, mais il ne s'agit que d'un aspect de la question.

En première ligne, par exemple, il y a la radio qui est peut-être la première source de victimisation. La nouvelle y est souvent diffusée avant même que les proches n'en aient été informés, et elle est lancée si rapidement sur les ondes qu'elle est incomplète, souvent erronée et surtout sans aucune contextualisation.

Dans la chaîne de l'information, suit le quotidien du matin, généralement un journal à très fort tirage, héritier direct du «penny journal» du début du siècle aux États-Unis, dont le contenu se compose surtout de nouvelles policières et de comptes rendus de matches sportifs. Photos de victimes et de scènes de crimes et gros titres y sont au moins aussi importants que le récit de la victimisation, lui-même généralement axé sur les faits les plus spectaculaires et rarement contextualisés. Le Journal de Montréal et le Journal de Québec, dont les ventes quotidiennes frisent le demi-million d'exemplaires, sont de ceux-là.

Le quotidien grand format, tels La Presse et Le Soleil, est généralement plus discret mais devient aussi généreux en titres, photos et récits sensationnels lorsqu'il s'agit d'une affaire qui passionne l'opinion, généralement un crime de violence particulièrement spectaculaire.

La télévision, au Québec et au Canada en général (ce n'est pas le cas aux États-Unis), se veut plus discrète mais couvrira tout de même elle aussi les crimes violents et spectaculaires qui soulèvent l'intérêt. On ne consacrera que très rarement plus de deux minutes à un tel sujet, le texte sera généralement discret et très bref, mais les images en apparence les plus sobres pourront avoir un effet traumatisant sur les victimes. Une photo de deux ambulanciers transportant une civière, en noir et blanc, dans un journal, n'a que peu d'impact. La même photo, grossie, sur l'écran d'une télécouleur, peut devenir 
insupportable pour les proches de la victime. Le gros plan d'une simple tache de sang, sur la chaussée, a sans doute plus d'impact à la télévision qu'une pleine page de photos dans le quotidien du matin.

Jusqu'à tout récemment au Québec, les crimes n'avaient été que très peu «exploités» par la télévision, et toujours dans le cadre des bulletins d'information. Depuis la rentrée de septembre 1989, les réseaux de Télévision Quatre-Saisons et de Télé-Métropole ont dans leur grille-horaire deux émissions exclusivement consacrées aux crimes: « 24 sur $24 »$ et «9-1-1». Il ne s'agit pas ici d'émissions d'affaires publiques, où l'analyse remplace l'anecdote alors que l'on traite d'un problème ou d'une forme de criminalité ou de victimisation de façon plus générale, en s'appuyant sur des témoignages de victimes ou d'intervenants. Ces deux émissions reprennent les crimes les plus sensationnels de la semaine en les illustrant de longues scènes des lieux et de photos et/ou d'entrevues avec les victimes ou leurs proches.

À l'instar des journaux hebdomadaires dits spécialisés (Allô Police, Photo Police), ces émissions reprennent ce que la radio et le quotidien du matin ont déjà exploité, mais en y ajoutant le maximum de détails et d'illustrations. Ce n'est d'ailleurs pas par hasard que les animateurs vedettes de ces deux émissions sont également au service d'un hebdomadaire spécialisé.

Chaque média ou catégorie de média a sa façon de traiter les victimes et peut représenter une source de victimisation particulière. C'est pourquoi nous nous référerons à l'ensemble des médias dans notre analyse tout en nous attachant plus spécialement aux quotidiens et aux hebdos spécialisés, sauf indication contraire.

Les émissions de fiction diffusent aussi de l'information sous le couvert du spectacle et sont sans doute ainsi à l'origine de plusieurs formes de victimisation. L'image qu'on projette de la police, des criminels et des victimes dans les séries télévisées et l'exploitation que l'on y fait de la victime dans les publications pornographiques peuvent sans doute ajouter aux souffrances des victimes de crimes el, surtout, être à l'origine de nouvelles victimisations; mais c'est là une tout autre problématique.

Il est vrai que la frontière entre la réalité et la fiction n'est pas toujours évidente dans ce type d'émissions mais ce sont les médias d'information, sauf exception, qui font ici l'objet de notre étude.

\section{LA VICTIME INSTRUMENTALE}

Avant d'analyser comment on traite la victime dans les médias, il importe d'essayer de saisir pourquoi on choisit certaines victimes plutôt que d'autres. 
Dans une étude que nous avons faite sur le sujet (partiellement publiée dans Parent, 1987), nous avons voulu démontrer que la sélection des nouvelles dans les médias était le fait de l'interaction entre la police et les médias, d'une complicité plus ou moins tacite entre policiers et journalistes, tantôt officielle, tantôt officieuse.

Les médias populaires, devenus entreprises commerciales très lucratives avec l'avènement du «penny journal», ont vite découvert au début du siècle jusqu'à quel point la couverture des crimes pouvait être rentable. Et les policiers volontaires d'alors ont saisi là l'occasion de faire carrière de protecteur du citoyen et de chasseur de criminels. Sous le couvert de conflits entre les deux groupes, policiers et journalistes profitent de cette complicité depuis:

Plus les policiers se sont faits les croisés de la lutte au crime, plus les journalistes ont découvert là un merveilleux filon, une matière première idéale pour la vente des journaux. Plus les journaux ont couvert le crime, plus les policiers ont pu obtenir d'effectifs et de pouvoir ... et plus les policiers sont devenus nombreux et puissants, plus ils ont pu produire de crimes... et plus les lecteurs des journaux ont craint le crime, plus on a pu vendre de journaux...

(Parent, 1987, p. 100)

Et pour boucler la boucle, il arrive que ce sont les crimes les plus spectaculaires, «la criminalité de sang», pour employer le mot de Foucault, que la clientèle des entreprises d'information réclame le plus, et que ce sont justement ces mêmes illégalismes qui sont les plus facilement solutionnés par les policiers...

Plusieurs auteurs ont bien étudié cette complicité, notamment Davis (1952), Schwoebel (1968), Fishman (1978), Tuchman (1978), Humphries (1981), Poveda (1982), Christiensen et al (1982), Smauss (1983), pour ne nommer que quelques classiques.

Les journalistes, choisissant la facilité, se contentent de plus en plus de l'information officielle transmise par des agents de communication spécialement formés et contrôlant l'information diffusée par les corps policiers.

Mais au centre de cette dynamique, il y a la victime, la victime qui devient l'instrument de ces deux agents de contrôle social que sont alors policiers et journalistes.

Ericson et al (1987 et 1989), dans leur analyse de l'interaction entre les médias et les divers agents de contrôle social dans la construction de la nouvelle, ont pu constater que la victime pouvait en devenir l'instrument: 
We observed many instances in which police officers went out of their way to encourage victims or relatives of victims to cooperate with reporters by giving interviews and still photographs that would publicize their plight.

(Ericson et al. 1989, p. 151)

Nous avons été témoin de scènes comme celles-là pendant 20 ans comme reporter ou chef de rédaction, surtout dans les cas d'homicide. Parfois un policier-enquêteur suggérera aux proches de recevoir tel ou tel journaliste, parfois il demandera aux proches une photo qui se retrouvera ensuite dans les médias, toujours dans le but d'aider à faire avancer l'enquête, à faire condamner l'infracteur ou celui non avoué de polir l'image de la police («There were also many explicity «hurrah» contexts in which the police sought to publicize their good work» (Ibid)... Et lorsqu' on recherche une victime disparue et qu'il faut vraiment publier sa photo et sa description dans les médias, on demande alors officiellement à la famille la permission de le faire, souvent à la suite de la signature d'une entente, comme dans les cas de disparition!

Que ce soit à la suite de l'intervention des policiers, à cause de la confusion qui suit immédiatement la victimisation ou pour faire entendre leur voix, les victimes perdent tout contrôle sur le récit de leur drame dès qu'elles collaborent avec les journalistes pour le rendre public. Cela fait partie de cette dépossession dont parle Baril (1980, p. 101):

Dès que l'une ou l'autre de ces agences intervient, la victime se voit dépouillée de ce qui était jusque-là sa tragédie personnelle. Désormais, l'affaire est publique. Selon nos interlocuteurs, le sentiment d'impuissance qu'ils ont vécu au moment de l'événement s'intensifie lors de l'intervention. Non seulement ils n'ont pas reçu l'aide qu'ils avaient espérée mais ils se sont sentis utilisés comme objets vers des fins qui n'était pas les leurs.

La «collaboration» avec les médias et la diffusion de la nouvclle font partie de la victimisation secondaire, au même titre que le sont souvent l'intervention policière et le processus judiciaire, mais avec plus d'éclat et sans qu'on puisse toujours en démontrer la nécessité.

L'information que transmettra la victime ou ses proches aux journalistes pourra souvent être plus traumatisante encore que celle que recueilleront les policiers:

Le renseignement du policier en effet est destiné à être étudié par les juges, analysé par les avocats, débattu oralement à l'audience. Il ne sera pas utilisé tel quel. Son intérêt ou son danger sera digéré par la procédure. 
Tout au contraire, le renseignement recueilli par le journaliste est une information à chaud jetée immédiatement à la face du public et bénéficiant d'une énorme diffusion. Que le renseignement soit faux, la situation est dès lors irréversible. Tous les démentis, rectifications, droits de réponse resteront inefficaces.

(Casamayor, 1975, p. 16)

\section{LA VICTIME CIBLE}

De plus en plus, on l'a dit, les journalistes se contentent de l'information officielle: c'est beaucoup moins coûteux pour l'entreprise que le journalisme d'enquête, et c'est sans aucun risque de poursuites pour le journaliste; mais i! arrive malgré tout que les journalistes frappent à la porte des victimes ou de leurs proches.

Nous insistons ici pour parler des proches, surtout dans les cas d'affaires de meurtre, mais il faut élargir le sens du mot victime de façon générale. L'Assemblée générale des Nations Unies (1985,p. 4) dit à ce sujet: «Le terme «victime» inclut aussi, le cas échéant, la famille proche ou les personnes à la charge de la victime directe et les personnes qui ont subi un préjudice en intervenant pour venir en aide aux victimes en détresse ou pour empêcher la victimisation.»

Très souvent, la victime ou ses proches feront l'objet d'un reportage, sur la scène même du crime, sans même s'en rendre compte.

Reporters spécialisés, surtout de la radio; cameramen et photographes sont aujourd'hui équipés de radio leur permettant d'être constamment branchés sur les ondes de police. À Montréal, les ordinateurs ont remplacé récemment la traditionnelle radio dans les voitures de police mais déjà cette difficulté d'accès a été contournée. Journalistes et photographes peuvent ainsi arriver sur les lieux des victimisations au même moment que les premiers patrouilleurs et très souvent avant les enquêteurs. Les quotidiens ont leur salle d'écoute des ondes de police et plusieurs journalistes sont alertés par les services de communication des grands corps policiers. Certains journalistes peuvent profiter de traitements de faveur et être avertis par certains enquêteurs, aussitôt l'appel reçu à l'escouade ou au poste (Parent, 1987; Gosselin et al., 1978).

Sur le coup de l'émotion, les victimes et leurs proches se feront photographier et parleront même à des journalistes sans s'en rendre compte ou sans savoir qui ils sont. Certains journalistes se tiendront constamment près des enquêteurs, se confondant littéralement avec eux. 
Des victimes et des proches s'adresseront souvent à des journalistes, croyant avoir affaire à des policiers. D’autres, impressionnés par le statut de vedette de certains star reporters, n'oseront pas leur refuser une entrevue.

Les journalistes, généralement, profitent de l'élément surprise et de la vulnérabilité de la victime dans les minutes qui suivent la victimisation. Et s'il y a réticence ou résistance de la part de la victime, le journaliste usera de toute une panoplie de moyens de pression. Ce poura être la complicité des policiers, comme on l'a vu précédemment, ou les menaces plus ou moins voilées: "vous faites mieux de me raconter ce qui s'est passé, sinon je raconterai ce qu'on m'a dit et ce ne sera pas à votre avantage... »; «vous me donnez une photo de la victime sinon je publie une photo d'elle gisant dans la rue...»; «ou nous publierons votre version des faits ou celle de la police ou de l'avocat de la défense...»!

Il arrive aussi qu'on troque les informations privilégiées sur le dossier qu'on a pu obtenir des policiers contre une entrevue avec la victime. D'autres victimes, enfin, se croient obligées de parler aux journalistes, de les recevoir. La chose est très fréquente dans les milieux moins favorisés, où les journalistes entrent plus facilement en contact avec les victimes afin de mener leur «enquête», enquête qui a surtout pour but d'obtenir des photos de famille et des lieux et qui consistera souvent en une entrevue de quelques phrases avec la victime et/ou ses proches. «Les hommes d'information, écrit le commissaire principal de police Berlioz (1982, p. 180), rechercheront la photo à sensation et le témoignage poignant: la volonté de frapper l'opinion est évidente. Entre nous, nous surnommons cette catégorie d'individus celle des nécrophages».

Il faut dire que les journalistes n'iront à peu près jamais frapper à la porte des victimes riches, plus scolarisées, dans les quartiers huppés. Ce sont là généralement des «victimes organisées», pour employer l'expression de Zauberman et Robert (1984, p. 46). Elles n’ouvriront généralement pas la porte aux journalistes ou leur opposeront un refus poli mais ferme, et cela souvent avec la complicité des policiers qui inciteront les journalistes à ne pas les importuner. Et lorsqu'une de ces victimes ouvrira sa porte aux journalistes, ou organisera une conférence de presse, ce sera dans le cadre d'une stratégie préparée par son avocat et/ou les policiers, comme dans les cas de kidnapping contre rançon.

L'irruption des journalistes sur la scène de la victimisation et leurs demandes peuvent donc représenter une première forme de victimisation secondaire qui vient s'ajouter à l'autre, dont les auteurs parlent surtout, qui consiste à voir sa photo, ses déclarations et des scènes rappelant la victimisation dans le journal ou au téléjournal du lendemain ou du soir même. 
Quant à la radio, on y diffuse la nouvelle au moment même où la victime est encore sur les lieux de la victimisation, en compagnie des policiers, le reporter envoyant ses premiers reportages en direct, assis dans sa voiture, grâce à sa quincaillerie spécialisée.

\section{LE PORTRAIT DE LA VICTIME}

Le premier contact entre journalistes et victimes peut être traumatisant mais c'est probablement le portrait qu'en feront les médias dans leur récit du crime qui constitue, croyons-nous, la source principale de victimisation secondaire par les médias. Dans le premier cas, il s'agira d'un mauvais moment à passer, un parmi d'autres, dans la confusion qui suit immédiatement la victimisation primaire: contacts avec les policiers, les voisins, les proches, au travail, à l'hôpital, etc. Le reportage, surtout écrit, et les images de la scène seront encore là, le lendemain ou une semaine plus tard, alors que la victime commencera à reprendre ses esprits et que s'entamera le processus de deuil, d'oubli, de cicatrisation.

Ce portrait de la victime devra être «bon vendeur» pour les entreprises commerciales que sont les journaux et les stations de radio ou de télévision, mais il procédera aussi d'une idéologie: le journaliste peut alors jouer le rôle d'agent de contrôle social en diffusant l'image du crime, du criminel et de la victime que le système policier et judiciaire lui donne. Il diffusera également l'image que se fait du criminel et de la victime son lecteur-acheteur, avec ses stéréotypes, ses préjugés et son ignorance.

Humphries (1981, p. 198), a constaté «that descriptions of offenders and victims were sparse, confined to sex, age, race and employment status, and that, with the exception of employment status, the pattern remained stable.»

Nous constatons avec lui que cette «typification» des victimes par les médias semble simpliste et simplifiante, faite de quelques mots, quelques catégories, mais c'est souvent cette simplification qui devient significative, avec tous les non dit qu'elle peut évoquer. L'agression d'un jeune chômeur de 18 ans et celle d'une veuve de 78 ans, sans qu'on en connaisse les circonstances, évoquent deux scénarios tout à fait différents.

Humphries (idem, pp. 202 et 203), qui a analysé la couverture des crimes par le New York Post sur une période de près de 20 ans a constaté, par exemple, qu'en 1951 on ne précisait pas si la victime et le criminel avaient ou non un emploi dans $80 \%$ des cas alors que la proportion était de $54 \%$ en 1968 .

Il suffit de s'y arrêter pour constater que dans les descriptions de victimes de crime que l'on retrouve dans nos médias, le fait d'avoir un emploi, être 
chômeur ou «sur le Bien-être social», célibataire, marié ou concubin, sont aussi importants que les variables d'âge et de sexe.

Souvent, non seulement s'attachera-t-on au statut ou à la situation de la victime mais on se référera souvent à son passé, selon que l'on veut polir ou ternir l'image de la victime. On parlera de l'ex-boxeur, de l'ex-barmaid, d'ex-prostituée, d'ancien dur à cuire; comme on pourra parler de l'ex-policier, l'ex-président de; l'ex-héros de guerre, l'ex-brave ceci ou cela...

Gosselin et al. (1978), Humphries (1981), Ericson et al. (1987 et 1989) et surtout Karmen (1984) ont particulièrement fait ressortir le vocabulaire classique que l'on retrouve dans les médias, surtout populaires, et qui charrie toujours les mêmes stéréotypes.

D'une part: l'innocente, la brave, la pauvre, la malheureuse et sans défense victime; d'autre part: le monstrueux, le sadique, le violent, l'agressif, le dangereux, le sinistre criminel! Gosselin et al (1978) ont ainsi répertorié, à même les titres et textes des reportages d'Allô Police et du Journal de Montréal, sur une période de 30 ans pour Allô Police et de 10 ans pour le Journal de Montréal, une trentaine de mots clés illustrant une certaine image de la victime et de l'auteur du crime, l'une en fonction de l'autre, la victime en fonction de la culpabilité et de la préméditation de l'infracteur.

Karmen (1984), qui a mené une étude semblable mais exclusivement au sujet des victimes, en arrive à cette conclusion:

Two types of sensationalism in the depiction of victims are found in media portrayals. The first involves understating the intensity of a victim's reaction to harm. The second type is a distortion of the opposite kind, in which the victimization experience is overstated or blown out of proportion to the actual damage done. Both understanding and overstating are forms of exploitation intended to take advantage of the victim's situation in order to serve some ulterior purpose.

(Karmen, 1984, p. 5)

Et l'auteur y va aussi d'exemples du vocabulaire utilisé dans chaque scénario.

Dans les cas de «Criminal Blaming in the Media» (idem, p. 117), les suspects sont des «ruffians», «pariahs», «the scum of the city», «litter of the streets», «debris», «parasites», «riffraff», «idle, menacing and strutting», «poor and unemployable», «on welfare and food stamps for doing nothing»... (lbid.) 
Dans l'autre scénario, "Victim Defending in the Media» (Idem, p. I18), les victimes sont décrites avec grande empathie: «hard working», «courageous», «envied because they were successful», «leading productive lives», «striving and loved», "deserving of more community and customer support», «believers in the American dream of capitalist free enterprise»... (Ibid.).

On saura le nombre et l'âge des enfants d'une victime mais rien sur la famille du suspect, à moins qu'il ne soit divorcé, séparé ou qu'il ne vive aux crochets de ses vieux parents. Par contre on saura que l'accusé a une Corvette, avec l'année et la couleur, surtout s'il est officiellement chômeur...

En sommes, on renvoie au lecteur-consommateur l'image qu'il a du criminel et de la victime: le public a-t-il cette image parce que les médias la lui projettent ou les médias la diffusent-elle parce que c'est celle que le public veut voir...? C'est l'histoire de la poule et de l'œuf!

\section{VICTIME COUPABLE OU NON COUPABLE}

Le traitement des victimes par les médias sera en bonne partie en fonction des typologies empiriques ou scientifiques qu'on fait ou qu'on a traditionnellement faites des victimes.

La classification de Fattah (Galaway et Hudson, 1981, p. 21) réflète assez bien les diverses typologies qui ont pu être faites depuis Von Hentig et Wolfgang: «Nonparticipating Victims», «Latent or Predisposed Victims», «Provocative Victims», «Participating Victims», «False Victims». La description de chacune de ces catégories de victimes variera dans les médias, selon l'image que les policiers voudront bien projeter mais aussi selon la représentation que s'en fera le journaliste et la perception qu'il aura de la représentation qu'en a son lecteur-acheteur.

Nous avons longuement parlé de l'image projetée dans les médias du «méchant criminel» et de la «bonne victime» mais il y a aussi la victime qui a «mérité de ce qui lui arrive».

Si la victime est un motard, un ex-détenu, un bagarreur, les médias se chargeront de le dire, souvent avec photo à l'appui (mug shot) accompagnant le casier judiciaire dans les archives de police. Et comme ces informations ne seront pas fournies par la victime ou ses proches, ce sont les policiers qui se chargeront de les diffuser.

Et comme ce genre de victimes sont impliquées dans les crimes où les policiers ont le moins de succès, les crimes les plus difficiles à résoudre (généralement des règlements de comptes entre gens criminalisés), ces derniers 
couvrent ainsi leur échec. Et qui fera pression sur les policiers pour découvrir le meurtrier ou le voleur d'un voleur de banque?

Quand il s'agit d'un meurtre d'enfant, d'un chauffeur de taxi ou du propriétaire d'un dépanneur, là où les policiers ont un excellent taux de réussite dans leur enquête, on jouera la carte de la victime innocente et du méchant tueur, donnant ainsi une image sécurisante et revalorisante du policier-gardien-de-la-paix.

Une «brave mère de famille» est assassinée par son mari, on la présente comme victime innocente. Une jeune femme est découverte assassinée, nue en bordure d'une route, on insistera pour rappeler qu'elle est divorcée, danseuse ou ex-danseuse, qu'elle fréquente les bars et le milieu de la drogue, qu'elle a un casier judiciaire, même s'il s'agit d'avoir troublé la paix ou de simple vagabondage...

Plusieurs chercheurs ont constaté depuis Sutherland que les criminels entretenaient systématiquement une image dévalorisante de leurs victimes et c'est ce que les policiers font des victimes à l'aide des médias lorsqu'ils se retrouvent devant des dossiers difficiles. Les homicides et autres crimes de violence nous viennent à l'esprit à ce sujet mais le silence que l'on garde sur les grandes fraudes, la criminalité d'affaire en général, tient aussi en bonne partie de la même logique.

S'il fallait établir une typologie des victimes les plus susceptibles d'être stigmatisées par les médias, ce serait probablement les mêmes qui sont les plus vulnérables à la victimisation primaire: «la victime coupable et la victime culturellement légitime», pour reprendre les catégories de Fattah (1980, p. 17). «La victime coupable», c'est celle que l'on blâme pour une culpabilité antérieure au crime («réelle ou imaginée», Ibid.) afin de se déculpabiliser: «la victime culturellement légitime», c'est celle qui appartient à «des personnes ou des groupes contre qui l'emploi de la violence est encouragé, toléré ou approuvé» (Idem, p. 19) dans une société donnée.

Même si on s'entend pour dire que la victimologie n'est plus centrée exclusivement sur l'infracteur, l'infraction et le rôle causal que la victime y tient, l'idée de la victime coupable a la vie dure. L'idéologie de la victimecatalysateur (victim precipitation) est toujours omniprésente, plus subtilement présentée qu'il y a 20 ou 30 ans, mais tout aussi pernicieuse.

Aucune typologie des victimes n'échappe à cette relation causale:

A victim typology cannot be an independant venture in the understanding of the victim, his relationship with his criminal, or his responsability in crime. 


\section{VICTIMES ET PRÉVENTION}

C'est également en vertu de ce principe (victim precipitation) qu'on bâtit les campagnes de prévention, les «community crime prevention», demandant aux victimes potentielles de prévenir leur propre victimisation, laissant ainsi croire qu'elles sont ou seront responsables de leur victimisation.

Certaines campagnes ont sans doute apporté quelques résultats, bien qu'il ne s'agisse souvent que d'un déplacement de cibles, mais les chercheurs sont généralement très critiques et sévères pour ces campagnes:

The idea that these strategies are oriented toward victimization prevention, not crime prevention, is borne out by their inability to control or prevent crime. There is no evidence that community crime prevention programs have significantly reduced crime, particularly serious violent crime.

(Timme et Norman, 1983, p. 64)

En plus de donner une explication facile à l'impuissance des «forces de l'ordre» à juguler la criminalité, ces campagnes de «responsabilisation» des victimes ont aussi pour effet de créer une autre diversion: invisibiliser les causes institutionnelles et socio-économico-politiques du crime:

The ideology of victim precipitation blames neither the structure of society nor the individual offender for crime. Instead it blames the victim who precipitates crime. Thus, the ideology of victim precipitation not only protects a criminogenic capitalist society currently in crisis from political criticism, but also legitimates a beleaguered and failing criminal justice system.

(Idem, p. 66)

Et dans toutes ces campagnes de prévention du crime, directement et indirectement, les médias jouent un rôle primordial, diffusant ainsi toute l'idéologie qui les sous-tend. Sauf de rares exceptions, des actions de quartier très limitées, il n'y aurait pas de campagnes de prévention du crime sans les médias.

Là où, sous prétexte de prévention et de "chasse aux criminels», l'information devient spectacle, un spectacle où la victime joue littéralement le rôle principal, ce sont ces émissions de télévision genre "Crime en direct». On y fait des reconstructions dramatisées de crimes non solutionnés, sur les lieux mêmes de ces crimes, où des comédiens professionnels jouent le rôle des victimes et des infracteurs. 
À Londres, la BBC présente une telle émission depuis juin 1984, "Crimewatch UK», une des émissions de télévision les plus regardées au Royaume-Uni, avec 15000000 de spectateurs (London Press Service). Chacune de ces émissions provoque entre 1000 et 3000 appels téléphoniques auxquels répondent des policiers en uniforme, en studio.

En Allemagne, une émission semblable, «Aktenzeiche X Y Ugelost», existe depuis 20 ans; aux États-Unis, le réseau NBC diffuse depuis 1987 l'émission «Unsolved Mysteries». Même la Hongrie a une émission du genre «Kek Fenny».

Quelques provinces canadiennes ont mis en ondes des émissions semblables et dans tous les cas, ce sont les policiers qui suggèrent les crimes à visibiliser, les réalisateurs y sélectionnant les victimisations qui se prêtent le plus à un scénario dramatique. On visibilise donc encore ici les crimes les plus spectaculaires et les plus "vendeurs», et les victimes et leurs proches peuvent ainsi assister à leur propre victimisation, revivre toutes les souffrances de cette victimisation «en direct»!

On peut prévoir que la télévision sera de plus en plus privilégiée par les policiers comme moyen de communication, comme c'est déjà le cas aux ÉtatsUnis. Ericson et al. ont constaté cette tendance et y voient deux raisons principales:

First, there was an appreciation that television can be more powerful in efforts at legitimation. The opposite of the literate and formal logic of print, television traffics in image and rhetoric. As such it has impact...

Second, there was an appreciation of the concomitant control qualities of broadcast news. Because its form works against its content broadcast news can be used to gloss over detail and reproduce occupational ideology. Even at the level of preparing the story broadcast news can be easier to manipulate. The brevity of items, use of fewer sources, and use of verbatim clips make broadcast news less subject to decontextualization than newspapers are.

(Ericson et al., 1989, p. 394)

\section{LA PEUR DU CRIME}

Un des effets de la survisibilisation des crimes dans les médias (une certaine criminalité surtout), que ce soit pour les décrire ou sous le prétexte de 
les prévenir ou d'assister les policiers dans leur enquête, est certainement la peur qu'elle peut engendrer.

Plus on diffuse une certaine image du crime, plus on fait peur... Et plus on fait peur, plus le public réclame des policiers et des pouvoirs pour le policier... Et plus il y a de policiers, plus on enregistre de crimes... Et plus il $y$ a de crimes, plus on en parle, plus on achète de journaux... Nous sommes encore ici plongés dans la relation complice médias-police.

On a dit et répété depuis le rapport Figgie (1980) que les médias alimentaient la peur du crime, peur plus diffuse que concrète, pour reprendre les mots de Brillon et Louis-Guérin (1983), une peur davantage créée par l'image de la criminalité diffusée dans les médias que par l'expérience personnelle de la victimisation.

La peur concrète par contre est une conséquence directe de la victimisation primaire, une forme de victimisation secondaire en ce qu'elle vient entacher la qualité de vie de la victime. Et elle semble omniprésente, surtout dans les cas de crimes de violence:

La violence provoque presque toujours une peur immédiate, ou même une panique, chez celui qui en fait l'objet...

(Baril, 1985, p. 32)

Parfois la peur est paralysante...

(Idem, p. 33)

La plupart des victimes de violence que nous avons rencontrées sont demeurées craintives et méfiantes après l'événement. Dans certains cas, nous les avons vues quelques années après l'agression et leurs craintes subsistaient toujours.

(Idem, p. 34)

D'autres victimes nous ont confié qu'elles avaient peur continuellement où qu'elles soient. Elles éprouvent une angoisse diffuse, une inquiétude qui n'a pas de motif précis.

(ldem, p. 37)

Ces résultats d'enquête nous autorisent, croyons-nous, à croire que le récit de la victimisation et les photos et images l'illustrant viendront ajouter à cette peur. De plus, il est légitime de croire que les crimes de violence relatés dans les médias viendront alimenter cette peur, transformant une source de peur diffuse en peur concrète. 
Il y a également la peur de représailles, bien présente dans les entrevues enregistrées par Baril (idem, p. 35), qui ne peut qu'être exacerbée par la diffusion du crime dans les médias, et il y a enfin la peur de devenir objet de curiosité, de ne plus pouvoir sortir dans la rue sans être reconnu et pointé du doigt, la peur d'être de nouveau victimisé. La peur engendrée par la publicité de la victimisation peut avoir diverses motivations:

D'autres, hommes et femmes, tenaient absolument à ce que les membres de leur famille ou leur employeur ou leurs amis ne soient pas informés, de peur de les inquiéter, par crainte d'être blâmés, stigmatisés, pénalisés. Chez l'une, le mutisme était dû à la fois aux menaces dont elle avait fait l'objet si jamais elle parlait à quiconque et à la peur d'être blâmée par ses enfants.

(Baril, 1984, p. 279)

«Fondée ou non, générale ou focalisée, la peur est l'émotion la plus communément exprimée par les victimes...», nous rappelle Baril (Idem, p. 223):

Tous les chercheurs qui ont interviewé des victimes de violence criminelle ont pris conscience du climat de peur dans lequel elles vivent...

(Idem, p. 225)

À notre avis, la peur d'être de nouveau victimisées ou le sentiment généralisé de crainte suscite ou accroît la plupart des autres traumatismes vécus par les victimes.»

(Idem, p. 228)

Au cœur de toute victimisation, il y a la peur, peur concrète, peur diffuse, peur d'avoir peur, peur qui «fait peur et engendre un cercle vicieux de comportements qui sont propres à favoriser le crime et, partant, la peur du crime» (Brillon, 1983, p. 14). Et au centre de ce cercle, il y a les médias qui alimentent cette peur.

\section{LES VAGUES DE CRIMES}

Les vagues de crimes sont un autre construit idéologique émanant directement de la relation medias-police qui a pour point de départ et pour cible la victime, ou plutôt des catégories de victimes, et qui peut être, croyons-nous, la source de victimisations multiples.

Plusieurs auteurs ont analysé ce phénomène qu'on pourrait ainsi décrire: les journalistes découvrent quelques crimes se ressemblant, on en fait état puis on parle d'une vague. Chaque jour, les médias recherchent des nouveaux 
cas, ce qui incite les policiers à en enregistrer davantage, les témoins à en déclarer plus et les victimes à rapporter des incidents qu'ils auraient tus en d'autres temps. Résultat: on crée un sentiment de peur généralisé. Sociologues, psychologues, criminologues et autres spécialistes s'emparent du phénomène et en font un problème social. Les procureurs sont plus alertes dans ce genre de dossier, les juges ont tendance à donner des sentences qu'ils veulent plus dissuasives et les politiciens s'emparent de la chose et concoctent des politiques ou des lois venant renforcer le contrôle social.

On serait peut-être tenté de croire, à l'instar de Baril (1982, p. 14) que «les victimes d'actes criminels constituent un marché peu rentable...», vu leur rôle «passif, peu glorieux, infâmant même» (Ibid.), ce qui toutefois ne nous semble pas correspondre à la réalité; mais lorsque les médias prennent comme cible un groupe ou une catégorie de victimes et en font l'objet d'une vague de crimes, l'entreprise apparaît vite éminemment rentable. Une vague de crimes peut fournir des reportages tous les jours, pendant des mois, puisque même l'absence de crimes peut tout autant être sujet d'une nouvelle que l'enregistrement de nouveaux crimes.

Fishman (1978) est, de tous les auteurs que nous avons consultés sur le sujet, celui qui, à notre avis, a le mieux analysé la construction des vagues de crimes, et cela à partir d'une pseudo-vague d'agressions contre des vieillards, à New York:

Crime waves are little more than the continued and heavy coverage of numerous occurrences which journalists report as a single topic... It presents a specific news event, or a number of such events, in terms of some broader concept.

(Fishman, idem, p. 534)

À New York, dans la recherche de Fishman, c'était les vieillards; à Montréal, nous avons eu droit, en 1984 et 1985, à une «vague» de meurtres d'enfants à partir de la disparition de trois enfants dont deux ont été découverts assassinés; plus récemment c'était une «vague» d'attentats contre les chauffeurs d'autobus... Dans tous ces cas, les statistiques ont démontré que les crimes enregistrés ne correspondaient absolument pas à la vague de crimes décrite dans les médias. Dans les cas des chauffeurs d'autobus de la STCUM, une recherche a même établi qu'il y avait eu moins d'attentats pendant la «vague» qu'au cours de la période correspondante l'année précédente!

Toutes les études sur les vagues de crimes que nous avons consultées (plus d'une vingtaine), depuis celles de Davis au Colorado (1952) jusqu'à Voumvakis et Ericson (1984) à Toronto, en arrivent à une même conclusion : l'augmentation du volume de reportages consacrés aux crimes de la vague 
ne correspond pas à une augmentation statistique équivalente de ces crimes et les vagues de crimes cessent le jour où les médias cessent d'en parler.

Quand on enregistre plus de crimes dans une région ou une ville, on parle d'augmentation de la criminalité; quand on attribue à un individu ou un groupe d'individus certains crimes, on parle de crimes en série; mais lorsque l'on parle d'une vague de crimes, on se réfère à un phénomène bien particulier; parfois à une certaine forme de criminalité, un lieu, un modus operandi mais la plupart du temps à une catégorie de victimes: vieillards, enfants, utilisateurs du métro, chauffeurs d'autobus ou de taxi, adolescentes, etc.

La vague de crimes, survisibilisant généralement des crimes de violence et exceptionnels, donne une image distordue des risques de victimisation, répand la peur chez les victimes cibles et restreint encore davantage ce que Baril (1984, pp. 326 à 329) appelle la «la marge de manœuvre» de la victime dans sa volonté de porter plainte ou non, la pression des proches, des témoins, policiers et procureurs devenant plus vive.

Plusieurs formes de victimisation peuvent donc être la conséquence directe de la construction des vagues de crimes qui, elles, ne sont possibles qu'en connivence avec les policiers:

While journalists may invent crime themes (I suspect the police suggest many of them), a crime wave needs enough incidents on the police wire to support it. The police have power both to veto and promote the media's construction of crime waves.

(Fishman, idem, p. 538)

\section{CONCLUSION}

Les médias, comme les policiers et l'appareil judiciaire, utilisent la victime à leurs fins. Ceux-là le font pour le maintien de l'ordre, la protection de la société; ceux-ci dans l'intérêt supérieur de la justice; les médias au nom du droit à l'information et en se posant comme les chiens de garde de la démocratie et de la justice.

Policiers, tribunaux et médias s'emparent de la victime, de sa victimisation et en font une plaignante, un témoin, un sujet d'information ou un objet de curiosité.

Sauf de rares exceptions, toutes dans «l'intérêt supérieur de la justice» ou de «la moralité publique» et rarement dans son intérêt à elle, la victime de crimes, celle-là qui fait le menu quotidien des médias d'information, est 
montrée, décrite, jugée sur la place publique alors qu'on traite de plus en plus l'infracteur derrière des portes closes.

Dans les médias plus qu'ailleurs, l'émergence et la reconnaissance des droits des accusés se sont faites hélas au détriment de la victime.

Vulnérable, sans défense, elle est livrée à la foule comme jadis on faisait parader le criminel dans les rues de la ville. En plus d'être l'instrument de cette interaction complice entre les médias et les autres agences de contrôle social, elle devient la vedette des scénarios accrocheurs qui font vendre des journaux et monter les cotes d'écoute des médias électroniques.

Faute de pouvoir accéder aussi facilement qu'avant l'avènement des chartes aux lieux et aux auteurs des victimisations, les médias se rabattent sur les victimes, une certaine catégorie de victimes, d'une certaine criminalité, de sang et de violence, qu'on retrouve dans les milieux surtout pauvres et défavorisés: là où les victimes sont le plus vulnérables, là où le spectacle de la victimisation peut faire le plus mal, mais là aussi où ce spectacle se vend le mieux!

La victimologie nouvelle et d'action a réussi chez nous à forcer certaines pratiques à tenir compte de la victime, à prévenir ou à atténuer le choc et les souffrances de la victimisation secondaire, sans tomber pour autant dans le militantisme vengeur et répressif. Elle a modifié les comportements à l'égard des victimes dans les grands corps de police, dans les cours de justice, et dans certaines autres bureaucraties gouvernementales, mais elle n'a pas encore réussi à toucher les pratiques des médias.

Dans un premier temps, les médias devront d'eux-mêmes apprendre que la victime n'est pas qu'objet de nouvelles et ne devient pas chose publique dès l'instant qu'elle est victime d'un crime dit de droit public.

Faute de droits spécifiques, ne devrait-on pas reconnaître au moins à la victime les mêmes droits qu'ont tous les autres citoyens : droit à la vie privée, droit à la dignité? À moins d'être homme ou femme publics-ques (et encore!), nul citoyen ne tolérerait qu'on fasse le récit de sa vie dans tous les médias et qu'on y fasse la manchette avec sa photo et sa maison sans son consentement. Et les tribunaux sont là pour assurer à tout citoyen honnête cette qualité de vie qui ne peut être que si elle est privée... Tout citoyen a droit à ce traitement jusqu'au jour où il devient victime de crime, surtout de ces crimes que policiers et médias ont intérêt à survisibiliser.

Être victime signifie souvent, dans l'état actuel des pratiques des médias. la perte de droits aussi fondamentaux que la sauvegarde de sa dignité, son honneur, sa réputation et sa vie privée... 
Un tel paradoxe ne saurait être encore longtemps entretenu! Le respect dû à la victime est une exigence maintenant incontournable et les médias ne pourront y échapper encore longtemps. Il y a là une certaine urgence dans les médias car, comme l'a écrit Laplante après avoir insisté sur la nécessité pour les médias de consentir à s'auto-évaluer, se surveiller et vérifier son évolution face au droit du public à l'information:

Si la presse n'amorce pas d'elle-même ces virages, ce qui malheureusement est parfois le cas, il faudra s'attendre à ce que les pouvoirs publics, toujours un peu lourdauds, passent à l'action. Il serait important que les médias comprennent d'eux-mêmes la portée de la nouvelle constitution, avant que le pouvoir judiciaire, qui ne comprend pas toujours parfaitement le rôle de la presse, leur impose de nouvelles règles. Car l'essentiel du rôle de la presse, le fondement même de l'information, c'est d'accroître la zone de liberté de ceux qui la consomment.

(Laplante, 1986, p. 48)

Il nous semble simpliste de croire que pourra cesser dans un avenir prévisible la diffusion des récits de victimisations et de l'identité de toutes les victimes.

On ne saurait renoncer aux procédures judiciaires inhérentes à une victimisation sous prétexte qu'elles viennent exacerber les souffrances de la victime et en ajouter de nouvelles. De la même façon on ne saurait garder secrètes les informations au sujet des victimes et des victimisations, et cela souvent dans l'intérêt même de la victime.

Rien ne s'oppose toutefois à ce qu'on civilise les pratiques dans les médias, tout comme on commence à le faire dans les cours de justice. Certains titres, certaines photos, certains détails scabreux n'ont pas plus leur pertinence dans les médias que les contre-interrogatoires agressants, et certaines preuves d'immoralité, de provocation et de caractère de la victime dans le processus judiciaire. L'éthique doit venir ici compenser pour les faiblesses de la loi et des chartes, qui semblent systématiquement refuser à la victime un traitement en tant que victime et des droits spécifiques.

Il faut aller au delà des débats sur le sensationnalisme («une notion avant tout morale», Sauvageau, 1986, p. 217) et situer cette problématique dans la perspective plus large et plus critique de l'analyse des fonctions réelles de l'information: faire éclater le discours sur les finalités des médias qui en soustendent les pratiques, les légitiment et en occultent souvent les mécanismes. Tant et aussi longtemps qu'on ne remettra pas en question, qu'on ne contestera pas les rôles et les fins que se sont toujours donnés et que se donnent encore les médias, la victime y sera considérée comme un objet de nouvelles au 
service du droit du public à l'information et un instrument de la liberté de presse.

Les médias chiens de garde de la démocratie; la publicité comme garantie de justice; le droit quasi absolu du public à l'information; l'objectivité du journaliste; le journalisme d'enquête; autant de fictions qui ne résistent pas à l'analyse des véritables finalités des médias comme entreprises commerciales et idéologiques.

Journalistes et médias ont accaparé le droit de définir ce qui est ou non d'intérêt public, comme les tribunaux celui de définir l'intérêt supérieur de la justice; et dans bien des cas, ce sont les victimes qui font les frais de cette interaction médias-police-tribunaux!

La victimisation secondaire, c'est peut-être davantage encore l'interaction entre toutes les agences qui s'intéressent à la victime que le fait de l'une ou l'autre d'entre elles...

\section{RÉFÉRENCES}

BARIL, M. (1980). «Ils n'ont plus la liberté: réactions à la victimisation et ses conséquences», Criminologie, vol. XIII, no 1.

BARIL, M. (1982), «Les laissés-pour-compte». Revue de la Sûreté du Québec, janvier 82.

BARIL, M. (1983). «Une illustration de la peur concrète : le cas des victimes», Criminologie, Vol. XVI, no 1.

BARIL, M. (1985). «Une nouvelle perspective: la victimologie», Traité de criminologie empirique: le phénomène criminel et la justice pénale au Québec, Montréal, P.U.M.

BARIL, M. (1984). «L'envers du crime», Les Cahiers de recherches criminologiques, no 2. Centre international de criminologie comparée.

BERLIOZ, J. M. (1982). "Police et mass media», Revue internationale de police criminelle, $37^{\mathrm{e}}$ année, no 360 .

BRILLON, Y. (1983). «La peur du crime et les tendances répressives du public envers les criminels», Revue internationale de criminologie et de police technique, vol. XXXVI, no 4.

BRILLON, Y. (1983). "La peur du crime et la punitivité chez les personnes âgées», Criminologie, vol. XVI, no 1.

BRILLON, Y. (1986). «L'opinion publique et les politiques criminelles», Criminologie, vol. XIX, no 1.

Canadian Criminal Justice Association (1985). Proceedings of the First National Seminar on Victim's Rights and the Judicial Process. Edited by Zambrowsky, J. et Davies, D. T., Toronto.

CASAMAYOR (1975). «La presse et l'activité judiciaire», Communication et Information, no 1 . 
CHRISTIENSEN, J.; SMITH, J.; HENDERSON, J. (1982). «The selling of the police: media, ideology and crime control», Contemporary Crisis, vol. 6, no 3.

COMMISSION DE RÉFORME DU DROIT (1987). L'accès du public et des médias au processus pénal, Document de travail 56 , Ottawa.

CONKLIN, J. E. (1981). Criminology, Macmillan Publishing Co. Inc., New York.

CONSEIL DE PRESSE DU QUÉBEC (1983). Réflexion sur les droits et responsabilités de la presse, Conseil de presse du Québec.

COTÉ G. et HODGINS, S. (1987). «État des connaissances de l'homicide», Cahier No 22, Institut Philippe Pinel de Montréal.

DAVIS, F. (1952). «Crime news in Colorado newspapers», American Journal of Sociology, 57.

DUPLÉ, N. (1986). «La liberté de la presse et la Charte canadienne des droits et libertés», Qu'est-ce que la liberté de presse?, Boréal.

ERICSON, E. V.; BARANEK, P. M.; CHAN, J. B. L. (1987). Visualizing Deviance a Study of News Organisation, University of Toronto Press.

ERICSON, E. V.; BARANEK, P. M.; CHAN, J. B. L. (1989). Negociating ControlA Study of News Sources, University of Toronto Press.

FATTAH, E. (1980). «Victimologie: tendances récentes», Criminologie, vol. XIII, no 1.

FISHMAN, M. (1978), «Crime Waves an Ideology», Social Problems, no 25, 531-543.

GALAWAY, B.; HUDSON, J. (1981). Perspectives on Crime Victims, The C. V. Mosby Company, St. Louis - Toronto - London.

GOSSELIN, L.; McFADDEN, E.; PILON, M.; TOUCHETTE, L. (1978). Violence et presse écrite, Office des droits des détenus de la Ligue des droits de l'homme.

GROUPE D'ÉTUDE FÉDÉRAL-PROVINCIAL CANADIEN SUR La justice pour les victimes d' actes criminels, (1983). Rapport. Ministre des Approvisionnements et Services. Ottawa.

HERJANIC, M. et MEYERS, D. A. (1976). «Notes on epidemiology of homicide in an urban area», Forensic Science, 8.

HUMPHRIES, D. (1981), «Serious crime, news coverage, and ideology.» Crime and Delinquency, 27.

KARMEN, A. (1984). Crime Victimes. An Introduction to Victimology, Brooks/Cole Publishing Company, California.

LAPLANTE, L. (1986). «La liberté de la presse, une liberté surveillée», Qu'est-ce que la liberté de presse?, Buréal.

LOUIS-GUÉRIN, C. (1983). «La peur du crime: mythes et réalités», Criminologie, vol. XVI, no ;1.

MERCY, J. A.; GOODMAN, R. A.; ROSENBERG, M. L.. et al. (1986). «Patterns of homicide victimization in the city of Los Angeles, 1970-1979", Bulletin of New York Academy of Medicine, 62.

PARENT, G.-A. (1987). «Presse et corps policiers : complicité et conflit», Criminologie, vol. $\mathrm{XX}$, no 1 .

POVEDA, T. G. (1982), «The FBI and domestic intelligence: technocratic or public relations triumph?», Crime and Delinquency, vol. 28, no 7.

SAUVAGEAU, F. (1978). «Liberté de la presse et droit du public à l'information: Fausse opposition ou contradiction véritable?», Communication et Information, vol. 2, no 3. 
SAUVAGEAU, F.; De BONVILLE, J. (1978), «Un code de déontologie pour la presse: difficultés d'élaboration et d'application», Communication et Information, vol. 2, no 3.

STATISTIQUE CANADA (1987). "L'homicide au Canada 1976-1985», ministère des Approvisionnements et Services, Ottawa, Canada.

TIMMER, D. A.; NORMAN, W. H. (1984). «The ideology of victim precipitation», Criminal Justice Review, vol. 9, no 2.

TUCHMAN, G. (1978). Making News : A study of the construction of reality, New York, Free Press.

VALLIÈRES, N.; SAUVAGEAU, F. (1981). Droit et journalisme au Québec, EDIGRIC/FPJQ.

VALLIÈRES, N. (1986). «La liberté de la presse dans la Charte québécoise», Qu'est-ce que la liberté de presse?, Boréal.

VOUMVAKIS, S. et ERICSON R. (1984). «News Accounts of Attacks on Women: A Comparison of Three Toronto Newspapers", Centre of Criminology, Université de Toronto.

WOLFGANG, D. E. (1958). Patterns in criminal homicide, Pennsylvania University Press.

ZAUMERMAN, R.; ROBERT, P. (1984). «Être victime et après?». Le pénal en première ligne et dernier ressort, Collection Déviance et contrôle social. Paris. 